\title{
Deterministic Chaos Concept and Invariance Problem in Discrete Dynamic Systems
}

\author{
V.A. Nikolsky \\ Transport and Telecommunication Institute, 1 lomonosova Street, Riga, LV-1019, Latvia \\ *Corresponding author: nv@tsi.lv
}

Copyright (C) 2013 Horizon Research Publishing All rights reserved.

\begin{abstract}
In given article new models of the discrete mappings are offered and on their basis the further development of the $K(E)$ transformation apparatus for discrete functions is given. On the basis of $K(E)$ transformation a method of discrete dynamic systems synthesis is set that in combination with principles of the classic theory of random processes makes it possible to carry out compensation (invariance) of the deterministic chaos, and optimal filtration of stationary random perturbation.
\end{abstract}

Keywords $K(E)$ transformation, invariance theory, deterministic chaos, automatic control, dynamic systems

\section{Introduction}

The design of discrete dynamic systems is often connected with seeking the principles of their construction and design, which include the complete or partial compensation for external perturbations $f_{n}$, as well as simulation of master controls $g_{n}$. The methods for the solution of this problem are different. In a number of practical cases we can distinguish some class of typical stimulus. In this regard, the problem of creation of automatic dynamic systems invariant to such external actions occurs. An efficient tool for achieving invariance may be the $K(E)$ transformation of lattice functions, developed by author during series of years. At present, the notion of $K(E)$ transformations covers the following typical cases:

(a) The form of the lattice function is determined.

(b) The function is not analytically determined a priori but is measurable on the observation interval.

(c) The function is random with known correlation functions.

(d) A priori information on the random function is absent.

(e) The function describes a quasideterministic signal.

Let us briefly explain the essence of the $K(E)$ transformation for these cases and then we expand this concept on for the deterministic chaos. The $K(E)$ transformation for discrete functions with a known analytical description is presented in [1]:

$$
K(E)=1+C_{0} E^{-1}+\ldots+C_{N} E^{-N-1} .
$$

For given lattice functions, the annihilating operator $K(E)$ is found in the form of a polynomial with respect to the shift $E^{-1}$ in such a way that $K(E) f_{n}=0$ or $f_{n}+C_{0} f_{n-1}+\ldots+C_{N} f_{n-N-1}=0$. Here, $n$ is the discrete time and $(n-i) \geq 0, C_{0}, \ldots, C_{N}$ are some constant coefficients.

Thus, the function $f_{n}$ can be interpreted as the solution to the homogeneous difference equation determined by the $K(E)$ transformation (case (a)).

If the description of the perturbation $f_{n}$ is unknown, the operator $K(E)$ is found as a result of the solution of the extrapolation problem connected with the function continuation found at the observation interval one step forward with the preservation of its analytical form [2] (case (b)).

For perturbations in the form of stationary random sequences with known correlation functions, the operator $K(E)$ is determined upon the solution of the optimal statistical prediction problem [3, 4] (case (c)).

If the correlation function of the random process is not known a priori, the determination of the $K(E)$ transformation is based on the application of self-learning algorithms [5] (case $(d))$.

When perturbation is a quasideterministic signal, the $K(E)$ operator is set at consideration of the system of difference equations, originating initial process (case of e). This result is represented in [6].

The analysis of the $K(E)$ transformations shows that, with increasing a priori uncertainty with respect to the signal, nonlinear algorithms play a more important role in the determination of the operator $K(E)$. The control system becomes nonlinear. The interest in nonlinear systems has another explanation. The understanding of the fact that some nonlinear differential and difference equations describing the behavior of the dynamic systems or signals acting in these systems, which do not contain random parameters in equations or initial conditions, can have chaotic solutions has 
been achieved in physics and technology in the recent years. As this fact was realized, the notion of a chaotic process was determined more precisely. At present, deterministic (dynamic) chaos [7-9] is mentioned more often, thus underlining the deterministic character of the noiselike signal behavior. Deterministic chaos (a quasistochastic process) possesses many properties of random oscillations: a continuous power spectrum, an exponentially decreasing correlation function, and an unpredictable character at large time intervals. Moreover, it has turned out that the nature of deterministic chaos is close to that of real processes $[8,10]$. The problem of invariance in this situation has been insufficiently studied [11]. The objective of this paper is the generalization of the notion of $K(E)$ transformation to the class of quasistochastic signals and the development of the method for the synthesis of discrete automatic systems invariant with respect to deterministic chaos based on this generalization.

\section{K(E) Transformation of Deterministic Chaotic Sequences}

The model of the perturbing signal $f_{n}$ is a nonlinear difference equation of the form:

$$
f_{n+1}=\Phi\left(h_{i}, f_{n}, f_{n-1}, \ldots, f_{n-N+1}\right) \quad i=\overline{1, k}
$$

at certain values of the initial conditions and external parameters $h_{i}$.

At present, the logistic map and the Hennon map [7], as well as some of its variants, have been extensively studied as discrete maps of form (2). Other important particular cases of Equation (2) can be the proposed relations of the form

$$
\begin{aligned}
& f_{n+1}=h\left(1-f_{n-1}^{2}\right) f_{n} \\
& f_{n+1}=h\left(1-f_{n} f_{n-1}\right) f_{n},
\end{aligned}
$$

where $h$ as beforeis the external parameter, and $n$ is the discrete time.

By changing the initial conditions and the external parameter $h$ in Equations (3) and (4), it is possible to obtain different scenarios of the transition to dynamic chaos. At present, there are no general mathematical methods for the explicit solution of such equations. The only available method of investigation is numerical experiments. Numerical simulation shows that the introduction of retardation into the logistic equation provides a variety of dynamic regimes, including oscillatory and quasistochastic ones.

Figures 1 and 2 show the iterations of Equation (3) and the projection of the phase trajectory onto the plane with the coordinates $f_{n}, f_{n+1}$, for $h=1.525$ and $f_{0}=f_{1}=0.5$, respectively.

Figure 3 shows an example of chaotic oscillations for model (3). Figure 4 shows the corresponding phase portrait in terms of the coordinates $f_{n}, f_{n+1}$ for $h=1.99, f_{0}=1.1$, and $f_{1}=0.5$.

The bifurcation diagram, respective to mapping (3) is shown on Figure 5, where external parameter $h$ changes across abscissa axis $(1.508<h<1.85)$ at initial conditions $f_{0}=$ 0 and $f_{1}=0.1$.

Figures $6\left(f_{n}\right.$ as a function of the number of steps) and 7 (phase portrait) illustrate the situation of the occurrence of chaotic oscillations for the map of model (4) for $h=2.2045$, $f_{0}=1, f_{1}=0.5$.

It can be seen from Figure 7 that the attractor resembles the known Lorentz strange attractor obtained upon the solution of the nonlinear system of three differential equations [12].

Figures 8 and 9 show the iterations of Eq. (4) and the projection of the phase trajectory onto the plane with the coordinates $f_{n}, f_{\mathrm{n}+1}$ for $h=2, f_{0}=0.6$, and $f_{1}=0.9$, respectively.

The map of form (1) and the corresponding particular cases can serve as the initial relations in the formation of the $K(E)$ transformation for quasistochastic signals.

Let us explain the method for obtaining $K(E)$ transformations using the logistic map (5) as an example:

$$
f_{n+1}=h\left(1-f_{n}\right) f_{n},
$$

where $h$ is the control parameter, and $f_{n}$ belongs to the interval $[0,1]$. By decreasing the index $n$ by unity in Equation (5) we write the system of two recurrence equations:

$$
\begin{aligned}
& f_{n+1}=h\left(1-f_{n}\right) f_{n} \\
& f_{n}=h\left(1-f_{n-1}\right) f_{n-1} .
\end{aligned}
$$

By determining the parameter $h$ from the second equation of system (6) and substituting its value into the first equation, we obtain (7)

$$
f_{n+1}=\frac{f_{n}\left(1-f_{n}\right)}{f_{n-1}\left(1-f_{n-1}\right)} f_{n} .
$$

This yields the following form of the sought operator $K(E)$ :

$$
K(E)=E-a,
$$

where $a=f_{n}\left(1-f_{n}\right) /\left(f_{n-1}\left(1-f_{n-1}\right)\right), E$ is shift operator symbol, which action is defined by relation $E \varphi_{n}=\varphi_{n+1}$.

Note that annihilating operator $K(E)$ as before possesses property:

$$
K(E) f_{n}=0, \quad K(E) \neq 0, f_{n} \neq 0 .
$$

The values of the $\mathrm{K}(\mathrm{E})$ transformations for some typical maps are given in the Table 1.

The aforementioned concept of $K(E)$ transformation for quasistochastic signals can serve as theoretical basis for synthesis of the discrete invariant automatic systems. 
Table 1. Major correspondences for quasistochastic signals

\begin{tabular}{|c|c|c|}
\hline $\mathrm{N}$ & Typical mapping & $K(E)$ transformation \\
\hline 1 & $f_{n+1}=r f_{n}-s f_{n}^{2}$ & $E-\frac{f_{n-1}^{2}\left(f_{n}-f_{n-1}\right)+f_{n} f_{n-2}\left(f_{n-2}-f_{n}\right)}{f_{n-1} f_{n-2}\left(f_{n-2}-f_{n-1}\right)}$ \\
\hline 2 & $f_{n+1}=h\left(1-f_{n-1}^{2}\right) f_{n}$ & $E-\frac{\left(1-f_{n-1}^{2}\right) f_{n}}{\left(1-f_{n-2}^{2}\right) f_{n-1}}$ \\
\hline 3 & $f_{n+1}=h\left(1-f_{n} f_{n-1}\right) f_{n}$ & $E-\frac{\left(1-f_{n} f_{n-1}\right) f_{n}}{\left(1-f_{n-1} f_{n-2}\right) f_{n-1}}$ \\
\hline 4 & $f_{n+1}=h\left(b-f_{n-1}^{2}\right) f_{n}$ & $E-\frac{f_{n}}{f_{n-1}}+\frac{\left(f_{n} f_{n-2}-f_{n-1}^{2}\right)\left(f_{n-2}^{2}-f_{n-1}^{2}\right)}{f_{n-1} f_{n-2}\left(f_{n-3}^{2}-f_{n-2}^{2}\right)}$ \\
\hline 5 & $f_{n+1}=h\left(b-f_{n} f_{n-1}\right) f_{n}$ & $E-\frac{f_{n}}{f_{n-1}}+\frac{\left(f_{n-2}-f_{n}\right)\left(f_{n} f_{n-2}-f_{n-1}^{2}\right)}{f_{n-2}^{2}\left(f_{n-3}-f_{n-1}\right)}$ \\
\hline
\end{tabular}

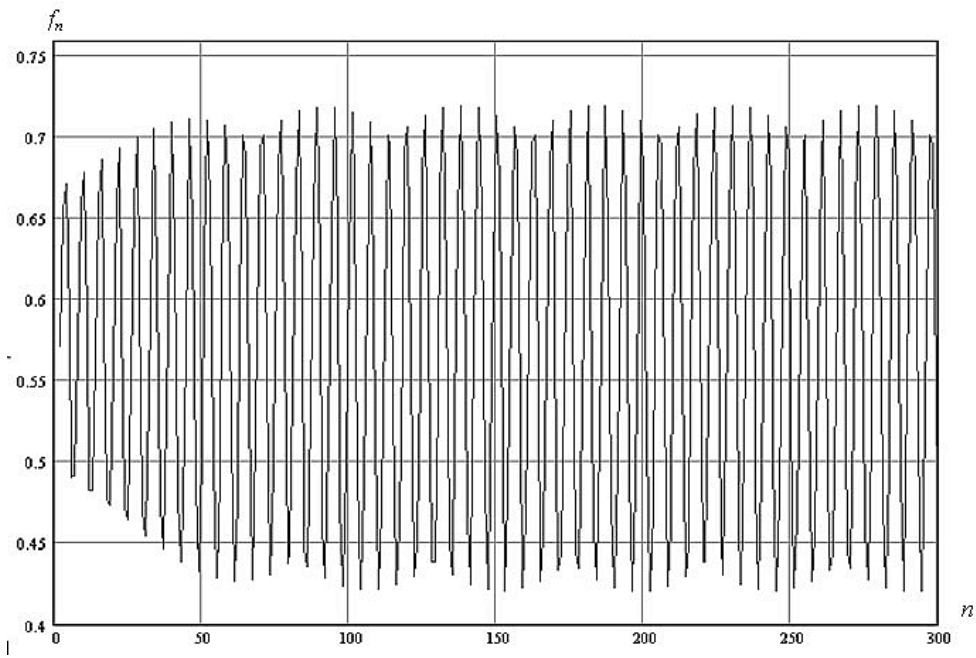

Figure 1. $\mathrm{f}_{\mathrm{n}}$ as a function of the number of steps for map (3): $h=1.525, f_{0}=f_{1}=0.5$

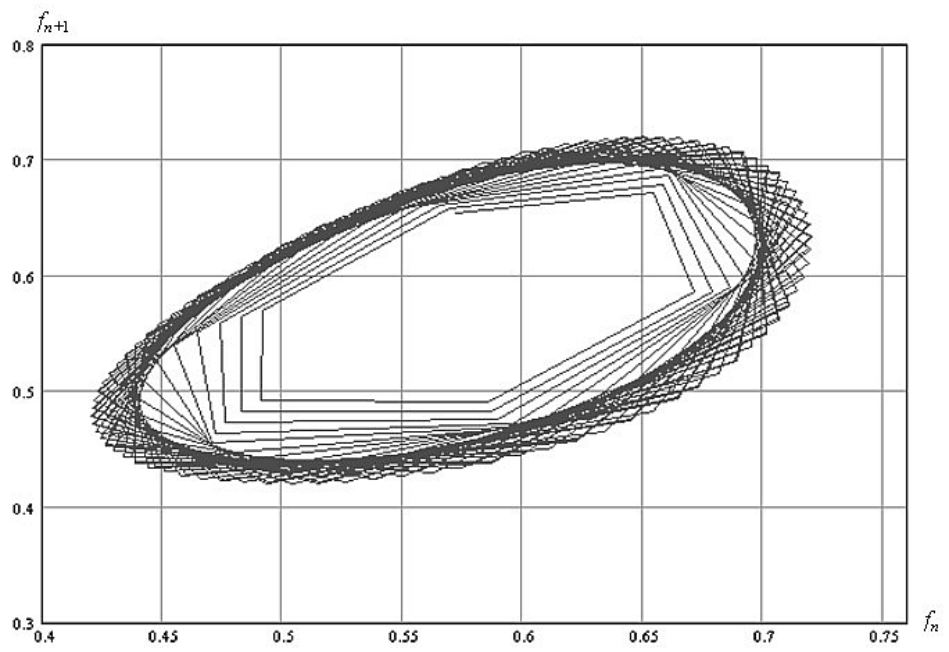

Figure 2. Phase portrait for map (3): $h=1.525, f_{0}=f_{1}=0.5$ 


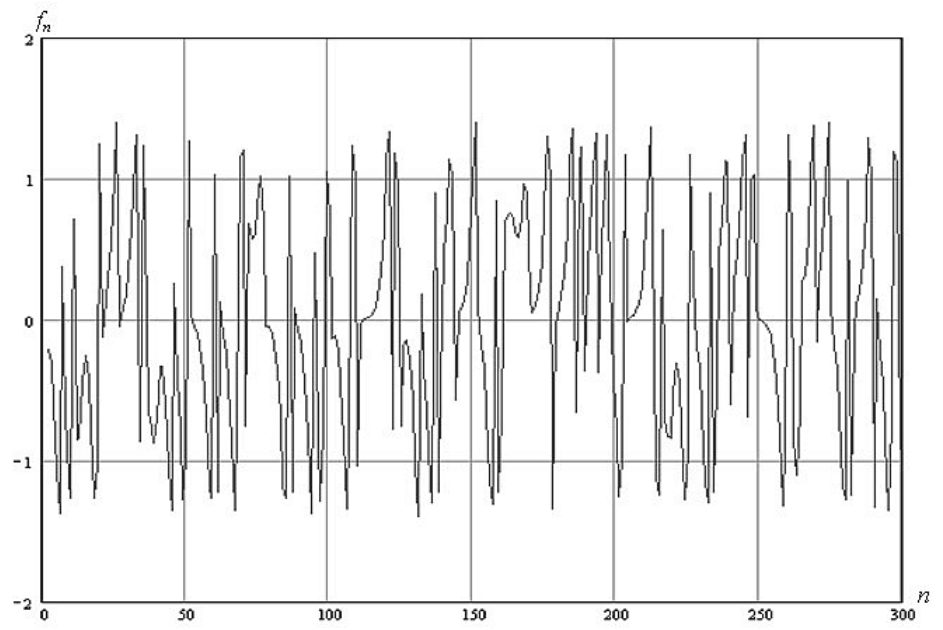

Figure 3. fnas a function of the number of steps for map (3): $\mathrm{h}=1.99, \mathrm{f} 0=1.1, \mathrm{fl}=0.5$

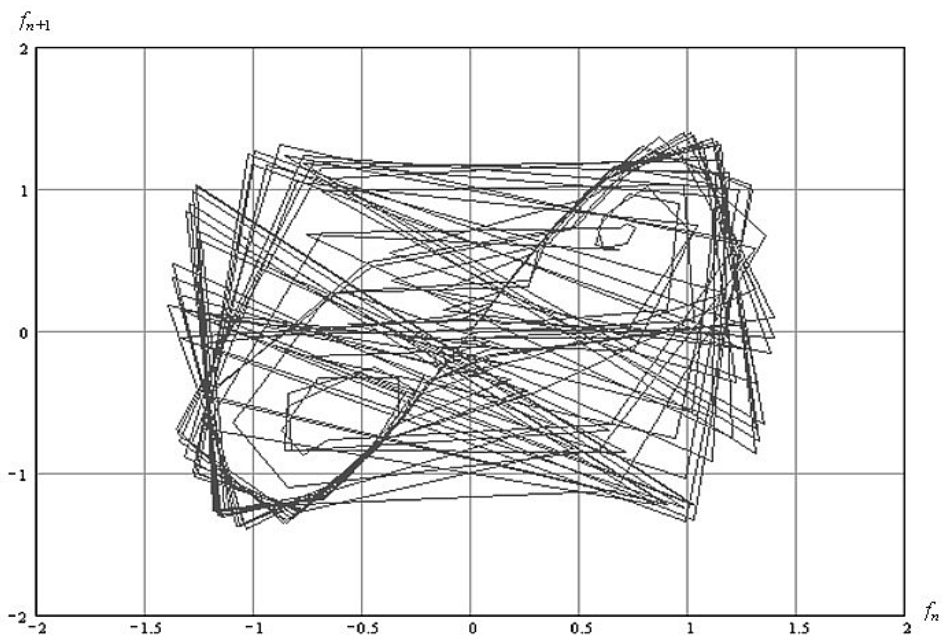

Figure 4. Phase portrait for map (3): $h=1.99, f_{0}=1.1, f_{1}=0.5$

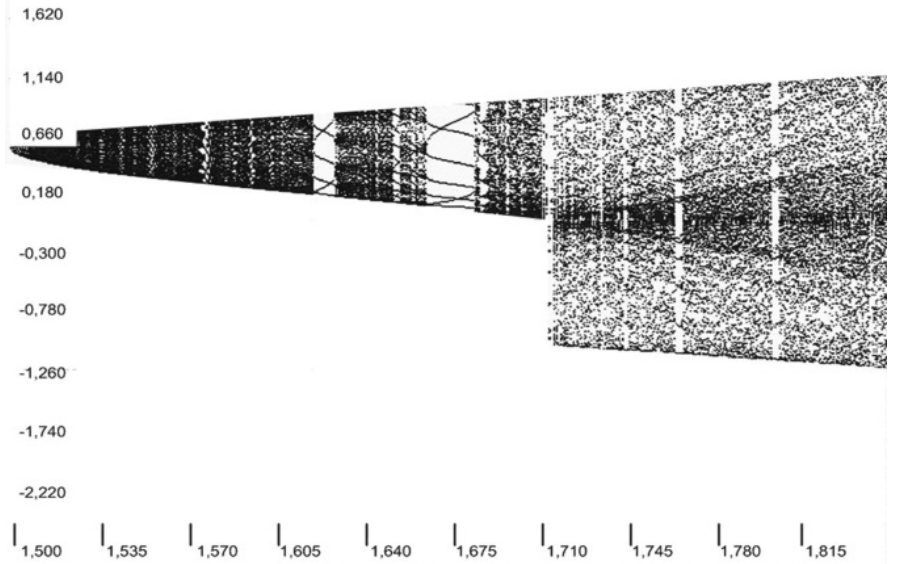

Figure 5. Bifurcation diagram, respective to discrete projection (3) 


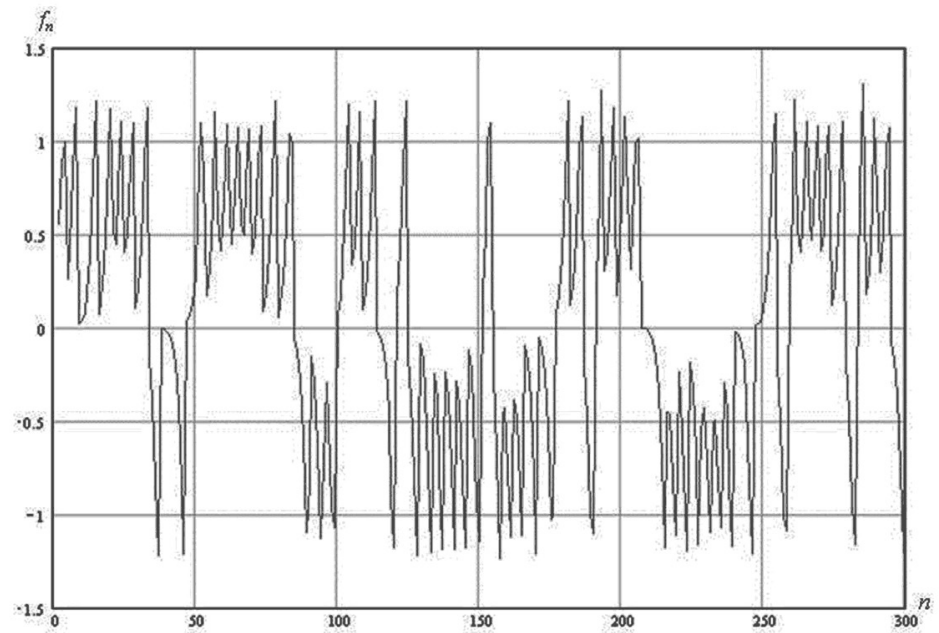

Figure 6. fnas a function of the number of steps for map (4): $\mathrm{h}=2.2045, \mathrm{f} 0=1, \mathrm{fl}=0.5$

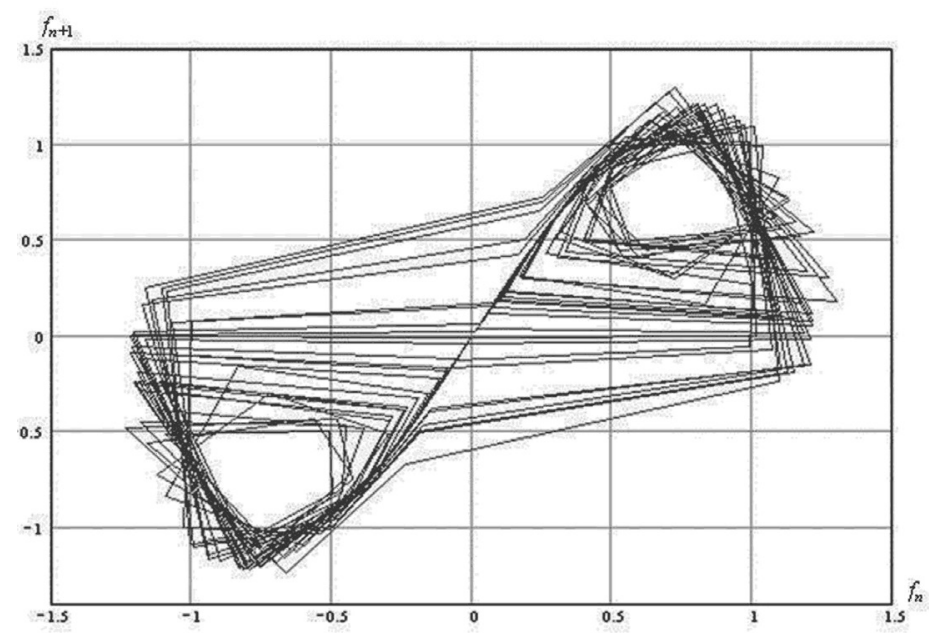

Figure 7. Phase portrait for map (4): $h=2.2045, f_{0}=1, f_{1}=0.5$

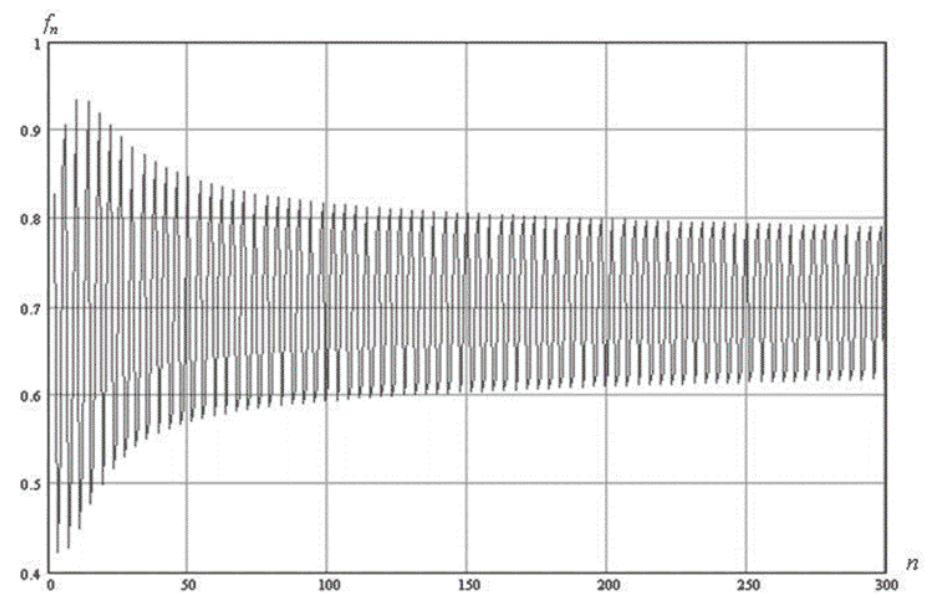

Figure 8. fnas a function of the number of steps for map (4): $\mathrm{h}=2, \mathrm{f0}=0.6, \mathrm{fl}=0.9$ 


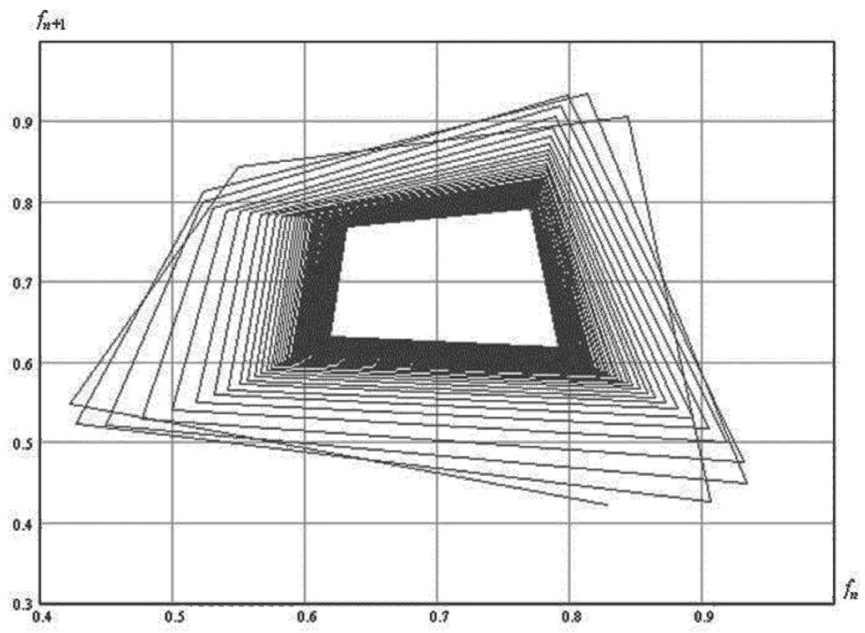

Figure 9. Phase portrait for map (4): $\mathrm{h}=2, \mathrm{f} 0=0.6, \mathrm{f} 1=0.9$

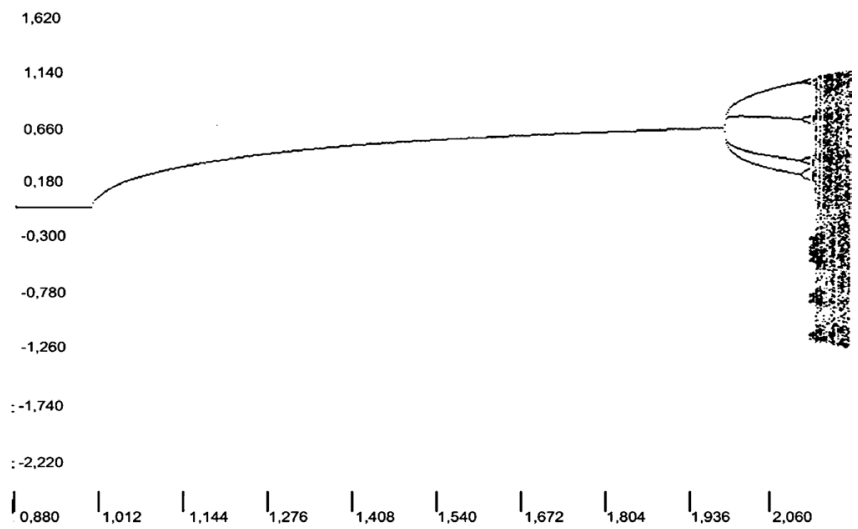

Figure 10. Bifurcation diagram, respective to discrete projection (4)

\section{Solution of the Discrete Stabilization System Synthesis Problem}

The structural diagram of the studied dynamic system is shown in Figure 11.

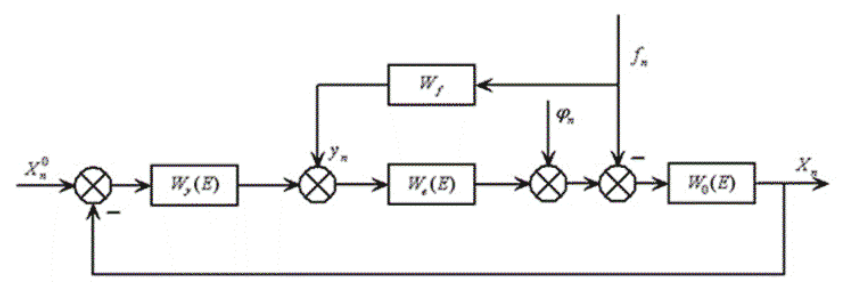

Figure 11. Structural diagram of the discrete stabilization system

The notation used in this figure is as follows:

$W_{0}(E)=\frac{R_{0}(E)}{Q_{0}(E)}$ is the transfer function of the control object written in the symbolic form;

$W_{e}(E)$ is the transfer function of the executive element; $W_{y}(E)$ is the transfer function of the control device;
$W_{f}=\frac{R_{f}}{Q_{f}}$ is the operator of the perturbation control channel; $X_{n}^{0}$ is the desired value of the controlled quantity; $f_{n}$ is the deterministic chaos (perturbing action); and $\varphi_{n}$ is the uncontrolled noise, which is taken in the form of a stationary random sequence.

The following problem is formulated: it is necessary to provide the finite duration of the transition process, zero established error caused by the quasistochastic sequence $f_{n}$, and the minimal dispersion of the filtering error caused by the stationary random noise $\varphi_{n}$ in the stabilization system. According to the method of $K(E)$ transformation, the solution of the synthesis problem includes two stages.

At the first stage, the transfer function of the control device is determined:

$$
W_{y}(E)=\frac{1}{K(E)} \frac{1}{K_{\varphi}(E)} \frac{M(E)}{P(E)} .
$$

Here, the factor $\frac{1}{K(E)}$ introduced in $W_{y}(E)$ for reproducing 
the initial program $X_{n}^{0}$ at the output of the system. In this case, the operator $K(E)$ is the $K(E)$ transformation of the analytically determined function $X_{n}^{0}$. The operator $\frac{1}{K_{\varphi}(E)}$ is included in $W_{y}(E)$ for filtering the random noise $\varphi_{n}$. The form of the operators $K(E)$ and $K_{\varphi}(E)$ is determined from tables [3].

The factor:

$$
\frac{M(E)}{P(E)}=\frac{M_{i} E^{i}+M_{i-1} E^{i-1}+\ldots+M_{1} E+M_{0}}{E^{q}+P_{q-1} E^{q-1}+\ldots+P_{1} E+P_{0}}
$$

is introduced in $W_{y}(E)$ in order to satisfy the condition of the finite time of the transition process of the system. The latter is achieved by equating to zero the coefficients of the characteristic equation of the closed system except for the coefficient at the highest power of $E$. The order of the polynomial $M(E)$ is determined as $i=\alpha+\gamma-1$, where $\alpha$ is the total order of the operators $K(E)$ and $K_{\phi}(E)$, and $\gamma$ and $q$ are the powers of the numerator and denominator of the transfer functions of the unchanged part of the system, respectively.

At the second stage, the transfer function of the perturbation control channel $f_{n}$ is determined:

$$
W_{f}=\frac{Q_{e}(E) Q_{y}(E)-A(E) K_{f}(E)}{R_{e}(E) Q_{y}(E)}
$$

where the operator $K_{f}(E)$ is introduced for compensating for the perturbation $f_{n}$.

The factor $A(E)=E^{1}$ provides the physical feasibility of the operator $W_{f}$, and $l=m-d$, where $m$ is the total power of the polynomials $Q_{y}(E)$ and $Q_{e}(E)$, and $d$ is the order of the operator $K_{f}(E)$. It will be assumed that the equations $R_{e}(E)=0$ and $Q_{y}(E)=0$ do not contain roots beyond the unit circle. If the expressions for errors with respect to the corresponding actions are determined, it can be easily seen that the presented synthesis method completely satisfies the above requirements.

Let us illustrate the proposed synthesis method using a numerical example.

Example.Let the transfer function of the control object be as follows: $W_{0}(E)=\frac{E}{E-1}$ for the scheme shown in Figure 11. The executive element performs retardation by one cycle. This yields that $W_{e}(E)=\frac{1}{E}$. The noise correlation function $R_{\varphi}(k)=\exp (-\theta|k|)$, where $k=0, \pm 1, \pm 2, \ldots, \theta$ is the damping parameter, and $K_{\varphi}(E)=E-e^{-\theta}$. The chaotic perturbation has a map of the form $f_{n+1}=h\left(1-f_{n} f_{n-1}\right) f_{n}, X_{n}^{0}=$ const . The task is to synthesize the invariant discrete stabilization system based on the $K(E)$ transformation for the given conditions.

The following operations should be executed for solving this problem.

Using (10), we determine the transfer function of the control device::

$$
W_{y}(E)=\frac{M_{1} E+M_{0}}{E-e^{-\theta}} .
$$

We find the transfer function of the open system:

$$
W_{y}(E)=\frac{R(E)}{Q(E)}=\frac{E\left(M_{1} E+M_{0}\right)}{(E-1)\left(E-e^{-\theta}\right) E} .
$$

We determine the characteristic polynomial of the closed system $N(E)$. Using the conditions of the finite duration of the transition process, we find coefficients $M_{0}$ и $M_{1}$ :

$$
N(E)=R(E)+Q(E)=E^{3}+b_{1} E^{2}+b_{2} E
$$

where

$$
b_{1}=M_{1}-e^{-\theta}-1, b_{2}=M_{0}+e^{-\theta} .
$$

By assuming $b_{1}=b_{2}=0$, we obtain

$$
M_{0}=-e^{-\theta}, M_{1}=1+e^{-\theta} .
$$

Taking into account formula (12), we determine the operator of the perturbation control channel:

$$
W_{f}(E)=\frac{E\left(a_{3}-e^{-\theta}\right)}{E-e^{-\theta}} .
$$

The parameter $a_{3}=\frac{\left(1-f_{n} f_{n-1}\right) f_{n}}{\left(1-f_{n-1} f_{n-2}\right) f_{n-1}}$ is found from Table 1. It follows from the formula for $W_{f}$ that the practical implementation of the invariance conditions is connected with the construction of the adaptive discrete filter. The structural diagram of adaptive filter,providing external perturbation compensation in discrete system for given mappingis shown in Figure 12.

We calculate the transfer function of the function with respect to the perturbation fn:

$$
\frac{x_{n, f}}{f_{n}}=\frac{\left(W_{f} W_{e}-1\right) W_{0}}{1+W_{0} W_{e} W_{y}}=\frac{a_{3}-E}{E} .
$$

We determine the component of the controlled quantity caused by the perturbation $f_{n}$ :

$$
x_{n, f}=\frac{a_{3}-E}{E} f_{n}=\frac{\left(1-f_{n-1} f_{n-2}\right) f_{n-1}}{\left(1-f_{n-2} f_{n-3}\right) f_{n-2}} f_{n-1}-f_{n} .
$$

It can be easily established using the annihilating property of the operator $K(E)$ that, after three cycles $x_{n, f}=0$. This equality holds only if $f_{n}$ is registered exactly.

We find the spectral density and variance of the system output with account for the noise action $\varphi_{n}$ :

$$
r(z)=\Phi(z) \Phi\left(z^{-1}\right) r_{\varphi}(z)=1-e^{-2 \theta},
$$

where $z$ is the variable of the discrete Laplace transform,

$$
\Phi(z)=\frac{W_{0}(z)}{1+W_{0}(z) W_{e}(z) W_{y}(z)},
$$




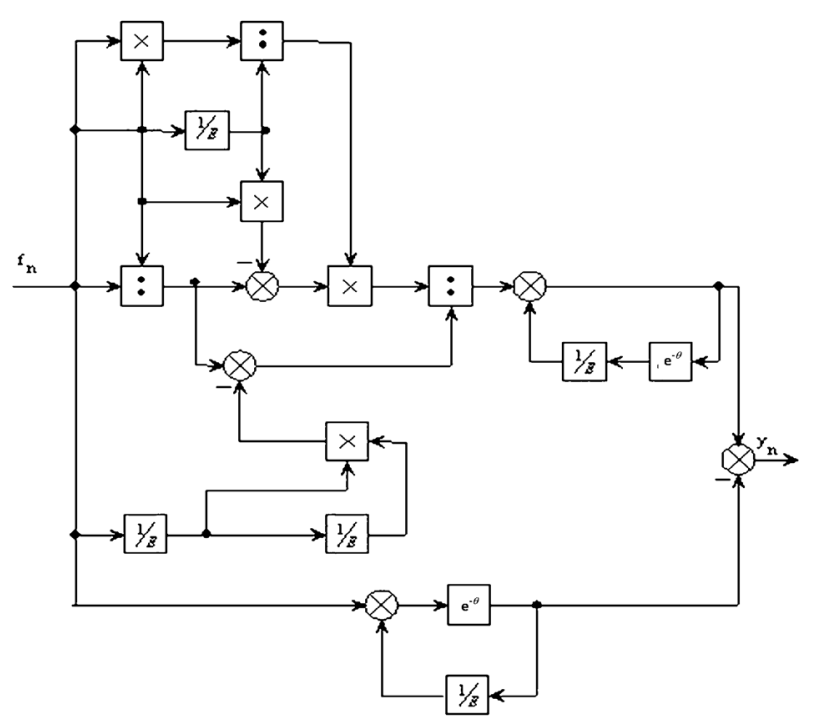

Figure 12. Structural diagram of the digital learning filter

and $\Phi(z)$ is the $z$ transfer function of the systemobtained from the operator $\Phi(E)$ if $E$ is replaced by $z$.

$$
r_{\varphi}(z)=\frac{\left(e^{-2 \theta}-1\right) z}{e^{-2 \theta} z^{2}-\left(1+e^{-2 \theta}\right) z+e^{-\theta}} .
$$

This yields the variance of the system output: $D=1-e^{-2 \theta}$. Thus, the established regime in the stabilization system is achieved in three cycles. The reaction of the system's output in the case of the action of deterministic chaos is equal to zero, and the optimal filtering of the random noise is performed as required.

\section{Numerical Experiment}

In order to verify the efficiency of the operation of the synthesized adaptive filter, a numerical experiment was performed for map (4) and the control parameter $h$ equal to 2 and 2.2045, respectively, and the initial conditions $f_{0}=0.6, f_{1}$ $=0.9$ and $f_{0}=1, f_{1}=0.5$, respectively. An example of discrete dynamic system responseto regular perturbation $f_{n}$ action is shown in Figure 13.

For the chaotic perturbation $f_{n}$, the results of the numerical experiment are shown in Figure 14.For the chaotic perturbation $f_{n}$, discrete dynamic systemresponse example is shown in Figure 14.
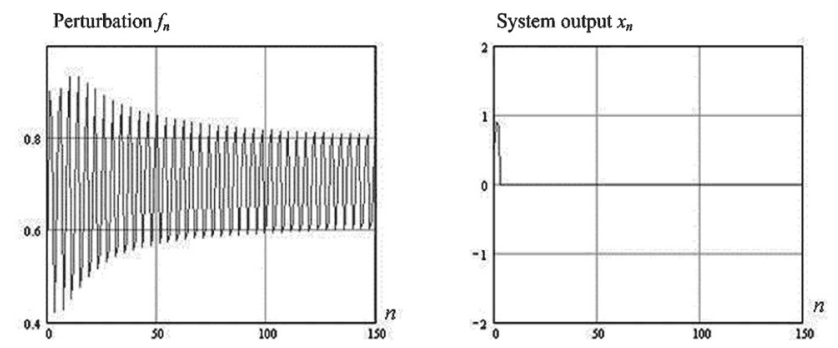

Figure 13. Variation of the perturbing action and the system output for the case of regular oscillations $f_{n}$
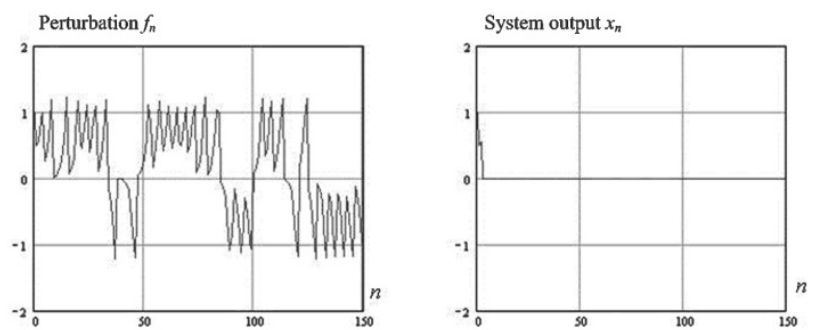

Figure 14. Variation of the perturbing action and the system output for the case of chaotic oscillations $f_{n}$

Thus the results of the numerical experiment prove the correctness of the theoretical concept concerning the compensation of the perturbing action both for the regular and chaotic oscillations. A solution of similar task for the system of the discrete combined management can be found in [11].

\section{Conclusion}

Certain perturbations in control systems can be interpreted as quasistochastic functions of discrete time.

It was demonstrated that the mathematical basis for the solution of the invariance problem in the case of deterministic chaos can be the $K(E)$ transformation for pseudorandom signals.

New models of the discrete mappings generating quasistochastic influences are offered. On the basis of these mappings the discrete functions $K(E)$ transformation apparatus further development was obtained. It made it possible to propose a method of synthesis of the discrete systems invariant to the deterministic chaos. In our opinion introduction of $\mathrm{K}(\mathrm{E})$ transformation for pseudorandom signals allows to solve also application-oriented tasks in the area information systems, in particular at synthesis of digital filters for communication systems.

Notice that the expounded researches, certainly, do not embrace all possible domains of $K(E)$ transformation applications. In particular, it can serve as theoretical basis of synthesis of generators and filters of nonlinear vibrations, at the analytical constructing of digital communication networks, and also at the solution of difference and differential-difference equations $[13,14]$.

\section{REFERENCES}

[1] V.A. Nikolsky, "Synthesis of Discrete Invariant Automatic Systems Using K(E) Transformation," Avtomatika i Vychislitel'naya Tekhnika, No. 4, 1969, pp. 47-52.

[2] V.A. Nikolsky, N.P. Sevastyanov, "K(E) Transformation of Lattice Functions in Discrete System Study Problems," In Automatics and Electromechanics, Moskow: Nauka, 1973.

[3] V.A. Nikolsky, N.P. Sevastyanov, "Perturbation Filtering 
Method in Discrete Control Systems Based on K(E) Transformation," Proceedings of 5th All_Union Meeting Invariance Theory and Its Application, Kiev: Naukova Dumka, 1979, pp. 337-346.

[4] V.A. Nikolsky, "Connection of Wiener Optimal Filtering Method for Invariance Principle Based on $\mathrm{K}(\mathrm{E})$ Transformation," VINITI Deposited Scientific Papers, No. 3,1986,

[5] V.A. Nikolsky, "Application of Combined Control Principle in the Problem of On Line Stocks Control," Ekonomiko Matematicheskie Metody, Vol. 16, No. 5, 1980, pp. 921-929.

[6] V.A. Nikolsky, "A Method of Step Control of Queue Length in Multiprocessor Numerical System with Variable Service Regime," Avtomatika i Vychislitel'naya Tekhnika, No. 6, 1998, pp. 61-70.

[7] G.G. Malinetskii, "Nonlinear Dynamics and Chaos", Moscow: KomKniga, 2006.

[8] Y.I. Neimark, P.S. Landa, "Stochastic and Chaotic Oscillations," Moscow: Nauka, 1997.

[9] V.A. Kravtsov, "Randomness as Predictability: the Concept of Partial Determinacy,“ Comm. Tech., Vol. 35, No. 1, 1990.

[10] G.Y. Riznichenko, A.B. Rubin, "Mathematical Models of Biological Production Processes, “ Moscow: Izd. Mosk. Univ., 1993.

[11] V.A. Nikol'skii, B.Ya. Tsil'ker, V.P. Pyatkov, "Synthesis of Tracking Combined Control Systems Operating in Conditions of Regular Chaos, “Avtomatika i Vychislitel'naya Tekhnika, No. 5, , 2009, pp. 45-52.

[12] J.C. Principe, L. Wang, M.A. Motter, "Local Dynamic Modeling with Self_Organizing Maps and Applications to Nonlinear System Identification and Control, "Proceedings of IEEE, 1998, Vol. 86, No. 11, pp. 2240-2258.

[13] V.A. Nikolsky, "To the question on equivalent transformations of linear heterogeneous difference equations with permanent coefficients, “ Differential equations, Vol. XI, No. 12, 1975, pp. 2278-2280.

[14] V.A. Nikolsky, "To the question on equivalent transformations of linear differential-difference equations with permanent coefficients, “ Differential equations, Vol. XX, No. 9, 1984, pp. 1638-1640. 